\title{
Identification of Human UDP-Glucuronosyltransferase and Sulfotransferase as Responsible for the Metabolism of Dotinurad, a Novel Selective Urate Reabsorption Inhibitor ${ }^{\text {S }}$
}

\author{
Koichi Omura, Keisuke Motoki, Seiichi Kobashi, Kengo Miyata, Katsuhiro Yamano, and \\ Takashi Iwanaga
}

Research Institute, Fuji Yakuhin Co., Ltd., Saitama City, Saitama, Japan (K.O., K.Mo., S.K., K.Mi., K.Y., T.I.)

Received September 15, 2020; accepted August 3, 2021

\section{ABSTRACT}

Dotinurad, a novel selective urate reabsorption inhibitor, is used to treat hyperuricemia. In humans, orally administered dotinurad is excreted mainly as glucuronide and sulfate conjugates in urine. To identify the isoforms of UDP-glucuronosyltransferase (UGT) and sulfotransferase (SULT) involved in dotinurad glucuronidation and sulfation, microsome and cytosol fractions of liver, intestine, kidney, and lung tissues (cytosol only) were analyzed along with recombinant human UGT and SULT isoforms. Dotinurad was mainly metabolized to its glucuronide conjugate by human liver microsomes (HLMs), and the glucuronidation followed the two-enzyme Michaelis-Menten equation. Among the recombinant human UGT isoforms expressed in the liver, UGT1A1, UGT1A3, UGT1A9, and UGT2B7 catalyzed dotinurad glucuronidation. Based on inhibition analysis using HLMs, bilirubin, imipramine, and diflunisal decreased glucuronosyltransferase activities by $45.5 \%, 22.3 \%$, and $22.2 \%$, respectively. Diflunisal and $3^{\prime}$-azido-3'-deoxythymidine, in the presence of $1 \%$ bovine serum albumin, decreased glucuronosyltransferase activities by $21.1 \%$ and $13.4 \%$, respectively. Dotinurad was metabolized to its sulfate conjugate by human liver cytosol (HLC) and human intestinal cytosol (HIC) samples, with the sulfation reaction in HLC sam- ples following the two-enzyme Michaelis-Menten equation and that in HIC samples following the Michaelis-Menten equation. All eight recombinant human SULT isoforms used herein catalyzed dotinurad sulfation. Gavestinel decreased sulfotransferase activity by $15.3 \%$ in HLC samples, and salbutamol decreased sulfotransferase activity by $68.4 \%$ in HIC samples. These results suggest that dotinurad glucuronidation is catalyzed mainly by UGT1A1, UGT1A3, UGT1A9, and UGT2B7, whereas its sulfation is catalyzed by many SULT isoforms, including SULT1B1 and SULT1A3.

\section{SIGNIFICANCE STATEMENT}

The identification of enzymes involved in drug metabolism is important to predicting drug-drug interactions (DDIs) and interindividual variability for safe drug use. The present study revealed that dotinurad glucuronidation is catalyzed mainly by UGT1A1, UGT1A3, UGT1A9, and UGT2B7 and that its sulfation is catalyzed by many SULT isoforms, including SULT1B1 and SULT1A3. Therefore, dotinurad, a selective urate reabsorption inhibitor, is considered safe for use with a small risk of DDIs and low interindividual variability.

\section{Introduction}

Dotinurad is a novel selective urate reabsorption inhibitor that was invented by Fuji Yakuhin Co., Ltd., who further codeveloped it with Mochida Pharmaceutical Co., Ltd., in Japan. Dotinurad was approved for the treatment of hyperuricemia by the Pharmaceuticals and Medical Devices Agency in January 2020. It exerts potent pharmacological effects and is also efficiently delivered to its target organ, i.e., the renal proximal tubule (Taniguchi et al., 2019; Omura et al., 2020). The major metabolites of dotinurad in humans were identified as glucuronide conjugate and sulfate conjugate, which are excreted via urine at $44.3 \%$ and

Financial support for the present study was provided by Fuji Yakuhin Co., Ltd. All authors are employees of Fuji Yakuhin Co., Ltd.

dx.doi.org/10.1124/dmd.120.000251.

S This article has supplemental material available at dmd.aspetjournals.org.
$20.0 \%$ of the dose, respectively, after oral administration (Omura et al., 2020).

Glucuronidation, one of the most important phase II metabolic reactions, plays a role in the detoxication of lipophilic molecules. It is catalyzed by UDP-glucuronosyltransferase (UGT), which has been classified into two families (UGT1 and UGT2) based on primary amino acid sequences. To date, 19 human UGT isoforms have been characterized (Meech et al., 2019). Sulfation is catalyzed by sulfotransferase (SULT) and is a well known phase II metabolic reaction for endogenous and exogenous substances. In humans, SULTs are classified into four families (SULT1, SULT2, SULT4, and SULT6), and 15 human SULT isoforms have been identified (Suiko et al., 2017).

The identification of enzymes involved in drug metabolism is important for predicting drug-drug interactions and interindividual variability. Some examples of DDIs via UGT have been reported in clinical studies; for instance, the effect of probenecid on the pharmacokinetics of acetaminophen (APAP) was investigated in healthy volunteers. Pretreatment

ABBREVIATIONS: APAP, acetaminophen; AZT, 3'-azido-3'-deoxythymidine; BSA, bovine serum albumin; DDI, drug-drug interactions; DTT, ( \pm )-dithiothreitol; HA, high affinity; HIC, human intestine cytosol; HKM, human kidney microsome; HLC, human liver cytosol; HLM, human liver microsome; HPLC, high-performance liquid chromatography; LA, low affinity; LC-MS/MS, liquid chromatography-tandem mass spectrometry; SULT, sulfotransferase; UGT, UDP-glucuronosyltransferase. 
with probenecid caused a decrease in APAP clearance $(6.23$ to $3.42 \mathrm{ml} /$ min per kilogram). Further, the urinary excretion of APAP glucuronide conjugate (348 to $74.5 \mathrm{mg}$ ) was reduced (Kamali, 1993). With respect to interindividual variability, it has been reported that toxicities in patients treated with irinotecan are caused by UGT1A1*28 polymorphisms (Takano and Sugiyama, 2017). For enzyme families with several isoforms, such as UGTs and SULTs, investigating whether one or more isoforms are involved in drug metabolism is essential. This is because the degree of DDI risk depends on the contribution ratio of the isoforms. Therefore, selecting an appropriate concomitant drug associated with a metabolic enzyme isoform can avoid or reduce the risk of DDIs. In addition, if the interindividual variability with respect to the activity of an enzyme that metabolizes a drug — mediated by a polymorphic enzymeis high, a dose adjustment will be required for patients expressing such a polymorphic enzyme.

Human isoforms of UGT and SULT responsible for the glucuronidation and sulfation of dotinurad have not been identified. For a more effective and safer use of dotinurad, we aimed to identify the human UGT and SULT isoforms responsible for the glucuronidation and sulfation of dotinurad to enable its safe use. Dotinurad glucuronidation was investigated in human tissue (liver, intestine, and kidney) microsomes and recombinant human UGT-expressing baculovirus-infected insect cells, and dotinurad sulfation was investigated in human tissue (liver, intestine, kidney, and lung) cytosol samples and recombinant human SULT-expressing Escherichia coli. Furthermore, for predicting DDIs, the contribution ratio of the enzymes involved in dotinurad metabolism is important. Therefore, kinetics and inhibition analyses using human liver and kidney microsomes and human liver and intestine cytosol samples were also performed.

\section{Materials and Methods}

Dotinurad (Fig. 1; Uda et al., 2020), dotinurad glucuronide conjugate, dotinurad sulfate conjugate (Fig. 1; supplemental data), and F12994 (internal standard) were synthesized by Fuji Yakuhin Co., Ltd. (Saitama, Japan). Adenosine $3^{\prime}$-phosphate $5^{\prime}$-phosphosulfate lithium salt hydrate, diflunisal, and bovine serum albumin (BSA) were purchased from Sigma-Aldrich (St. Louis, MO). 3'-Azido3'-deoxythymidine (AZT) was purchased from Sigma-Aldrich and CombiBlocks (San Diego, CA). Imipramine hydrochloride, bilirubin, ( \pm )-dithiothreitol, and salbutamol sulfate were purchased from Wako Pure Chemical Industries, Ltd. (Osaka, Japan). Gavestinel was purchased from Tocris Bioscience (Bristol, UK). Pooled human tissue microsomes (liver, 50 individuals including males and females; intestine, 13 individuals including males and females; kidney, 12 individuals including males and females) and human tissue cytosol (liver, 50 individuals including males and females; intestine, 13 individuals including males and females; kidney, four individuals including males and females; lung, four individuals including males and females) were purchased from Sekisui XenoTech, LLC (Kansas City, KS). UGT cofactor mixture A (containing $25 \mathrm{mM}$ UDP-glucuronic acid in water), UGT cofactor mixture B (containing $250 \mathrm{mM}$ Tris- $\mathrm{HCl}$, $40 \mathrm{mM} \mathrm{MgCl}$, and $0.125 \mathrm{mg} / \mathrm{ml}$ alamethicin in water), and recombinant human UGTs (UGT1A1, UGT1A3, UGT1A4, UGT1A6, UGT1A7, UGT1A8, UGT1A9, UGT1A10, UGT2B4, UGT2B7, UGT2B10, UGT2B15, and UGT2B17) expressed in baculovirus-infected insect cells were purchased from Corning (Woburn, MA). E. coli expressing recombinant human SULTs (SULT1A1*1, SULT1A2, SULT1A3, SULT1B1, SULT1C2, SULT1C4, SULT1E1, and SULT2A1) was purchased from
Cypex Ltd. (Scotland, UK). All other reagents were of the highest commercially available grade.

Glucuronidation of Dotinurad. A typical incubation mixture (200 $\mu \mathrm{l})$ contained $50 \mathrm{mM}$ Tris- $\mathrm{HCl}$ ( $\mathrm{pH} 7.5$ ), 8 mM MgCl $2,2 \mathrm{mM}$ UDP-glucuronic acid, 25 $\mu \mathrm{g} / \mathrm{ml}$ alamethicin, and $50 \mu \mathrm{M}$ dotinurad with $0.5 \mathrm{mg} / \mathrm{ml}$ human tissue microsomes (liver, intestine, and kidney) or $0.25 \mathrm{mg} / \mathrm{ml}$ recombinant human UGTs. Dotinurad was dissolved in DMSO, and the final concentration of DMSO in the reaction mixture was $1 \%(\mathrm{v} / \mathrm{v})$. After preincubation at $37^{\circ} \mathrm{C}$ for 5 minutes, the reactions were initiated by the addition of microsomes. The reaction mixtures were incubated at $37^{\circ} \mathrm{C}$ for 30 [human liver microsomes (HLMs) and recombinant human UGTs] or 60 minutes [human intestine microsomes (HIMs) and human kidney microsomes (HKMs)], and the reaction was terminated by adding $100 \mu \mathrm{l}$ of ice-cold 4\% (v/v) acetic acid acetonitrile containing F12994 as an internal standard. The protein concentration and reaction time for each tissue microsomal assay were optimized based on linearity, in advance. To each terminated reaction mixture, $1 \mathrm{ml}$ of distilled water was added, and samples were then stirred. Standard curves were prepared as described previously herein, except that incubation was not included and glucuronide conjugate standards were used instead of dotinurad.

Kinetic Analysis of Glucuronidation in HLMs and HKMs. Kinetic studies were performed using pooled HLMs and HKMs. Glucuronosyltransferase activities in the presence of dotinurad (concentrations ranging from 5 to 500 $\mu \mathrm{M})$ were determined. The kinetic parameters were estimated as follows from the fitted curves using the Michaelis-Menten equation or the two-enzyme Michaelis-Menten equation, using WinNonlin (version 6.4; Pharsight, Mountain View, CA).

The Michaelis-Menten equation is as follows:

$$
v=V_{\max } \times[S] /\left(K_{m}+[S]\right),
$$

where $v, V_{\max },[S]$, and $K_{\mathrm{m}}$ are the rate of reaction, maximum velocity, substrate concentration, and Michaelis-Menten constant, respectively. The two-enzyme Michaelis-Menten equation is as follows:

$$
\begin{aligned}
v= & \left\{V_{\max _{H A}} \times[S] /\left(K_{m_{H A}}+[S]\right)\right\} \\
& +\left\{V_{\max _{L A}} \times[S] /\left(K_{m_{L A}}+[S]\right)\right\},
\end{aligned}
$$

where the subscripts $H A$ and $L A$ represent the high- and low-affinity components, respectively. The best fit was based on the Akaike information criterion.

Inhibition Analysis of Glucuronidation in HLMs and HKMs. Bilirubin, imipramine, diflunisal, and AZT were tested for their inhibitory effects on dotinurad glucuronidation in pooled HLMs and HKMs (only diflunisal and AZT were used). Bilirubin is a well known typical substrate, and it was used for the inhibition analysis of UGT1A1 (Yamanaka et al., 2007; Shiraga et al., 2012). Imipramine was used for the inhibition analysis of UGT1A3 and UGT1A4 (Yamanaka et al., 2007; Shiraga et al., 2012). Diflunisal was used for the inhibition analysis of UGT1A9 (Walsky et al., 2012). AZT is a well known substrate of UGT2B7 (Court et al., 2003; Yasuda et al., 2011). Bilirubin, imipramine, diflunisal, and AZT were dissolved in DMSO, and their concentrations in the reaction mixture were adjusted to $10,100,50$, and $1000 \mu \mathrm{M}$. Glucuronosyltransferase activities were determined at $50 \mu \mathrm{M}$ concentrations of dotinurad in a manner similar to that described previously herein.

Kinetic and Inhibition Analysis of Glucuronidation in HLMs and HKMs with 1\% BSA. In this study, a typical incubation mixture $(200 \mu \mathrm{l})$ for kinetic analysis contained $50 \mathrm{mM}$ Tris- $\mathrm{HCl}(\mathrm{pH} 7.5), 8 \mathrm{mM} \mathrm{MgCl} 2,2 \mathrm{mM}$ UDP-glucuronic acid, $25 \mu \mathrm{g} / \mathrm{ml}$ alamethicin, $1 \% \mathrm{BSA}$, and dotinurad (at concentrations ranging from 5 to $500 \mu \mathrm{M}$ ) containing $0.5 \mathrm{mg} / \mathrm{ml}$ HLMs or HKMs. After preincubation at $37^{\circ} \mathrm{C}$ for 5 minutes, the reactions were initiated by the

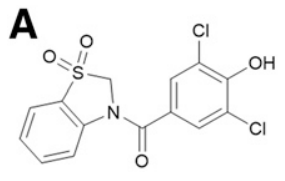

B

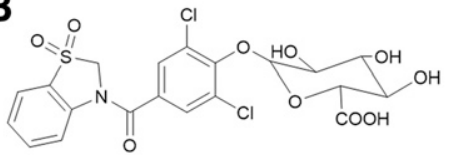

C

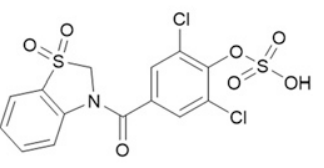

Fig. 1. Chemical structure of dotinurad (A), its glucuronide conjugate (B), and its sulfate conjugate $(\mathrm{C})$. 
addition of microsomes and incubated at $37^{\circ} \mathrm{C}$ for 30 (HLMs) or 60 minutes (HKMs) and were then terminated by adding $100 \mu \mathrm{l}$ of ice-cold $4 \%$ (v/v) acetic acid acetonitrile containing F12994 as an internal standard. To each terminated reaction mixture, $1 \mathrm{ml}$ of distilled water was added, and then samples were stirred.

Diflunisal and AZT were evaluated for their inhibitory effects on dotinurad glucuronidation in pooled HLMs and HKMs with $1 \%$ BSA, and their concentrations in the reaction mixture were adjusted to 500 and $1200 \mu \mathrm{M}$. Glucuronosyltransferase activities were determined at $50 \mu \mathrm{M}$ (HLMs) or $150 \mu \mathrm{M}$ (HKMs) concentration of dotinurad in a manner similar to that described previously herein.

LC-MS/MS Analysis of Dotinurad Glucuronide Conjugate. The glucuronide conjugate in the reaction mixtures was quantified by LC-MS/MS using a 1100 series high-performance liquid chromatography (HPLC) system (Agilent Technologies, Inc., CA) and API3000 (AB SCIEX, Framingham, MA). The terminated reaction mixtures were processed using solid-phase extraction in a 96well plate format. The glucuronide conjugate was eluted with $100 \mu l$ acetonitrile from the solid-phase and was diluted twice with distilled water. In total, $2 \mu 1$ of the processed reaction mixture was injected into an LC-MS/MS. Dotinurad glucuronide conjugate and matrix constituents in the reaction mixture were separated using an Inertsil ODS-3 $(2.1 \times 150 \mathrm{~mm}, 3 \mu \mathrm{m}$; GL Sciences, Tokyo, Japan) at $50^{\circ} \mathrm{C}$ with a mobile phase of $5 \mathrm{mM}$ ammonium acetate $(\mathrm{pH} 4)$ prepared in water and methanol $(50: 50, \mathrm{v} / \mathrm{v})$. The total flow rate was set at $0.2 \mathrm{ml} / \mathrm{min}$. Ionization was conducted in turbo ion spray and negative ion modes. Dotinurad glucuronide conjugate was analyzed as $[\mathrm{M}-\mathrm{H}]^{-}$ions in the multiple reaction monitoring mode (transitions: dotinurad glucuronide conjugate 533.8/357.9 and internal standard F12994 340.8/144.9). For the reaction mixtures in the inhibition study, the mobile phase ratio was changed to wash the inhibitors, as described below. The initial mobile phase was $50 \% 5 \mathrm{mM}$ ammonium acetate $(\mathrm{pH} 4)$ in water and $50 \%$ methanol. The percentage of methanol was increased to $95 \%$ at 15 minutes and maintained at $95 \%$ at $15.1-28$ minutes. From 28.1 to 38 minutes, the column was re-equilibrated with $50 \%$ methanol. The concentration range of the standard curve of glucuronide conjugate was between 50 and 30,000 nM. However, LC-MS/MS, using a Shimadzu Nexera HPLC system (Shimadzu Corporation, Kyoto, Japan) and QTRAP4500 (AB SCIEX), was used for "kinetic and inhibition analysis of glucuronidation in HLMs and HKMs with $1 \%$ BSA." The injection volume was changed from $2 \mu l$ to $0.5 \mu l$ in this case.

Sulfation of Dotinurad. A typical incubation mixture $(200 \mu \mathrm{l})$ contained $100 \mathrm{mM}$ potassium phosphate buffer ( $\mathrm{pH} 7.4$ ), $10 \mathrm{mM} \mathrm{MgCl} 2,1 \mathrm{mM}$ ( \pm )-dithiothreitol, $30 \mu \mathrm{M}$ adenosine $3^{\prime}$-phosphate $5^{\prime}$-phosphosulfate lithium salt hydrate, and $50 \mu \mathrm{M}$ dotinurad with $0.5 \mathrm{mg} / \mathrm{ml}$ human tissue cytosol samples (liver, kidney, and lung), $0.1 \mathrm{mg} / \mathrm{ml} \mathrm{HIC}$ samples, $0.01 \mathrm{mg} / \mathrm{ml}$ recombinant human SULTs (SULT1A2, SULT1A3, SULT1C4, and SULT1E1), $0.05 \mathrm{mg} / \mathrm{ml}$ recombinant human SULTs (SULT1A1*1 and SULT1B1), or $0.1 \mathrm{mg} / \mathrm{ml}$ recombinant human SULTs (SULT1C2 and SULT2A1). Dotinurad was dissolved in DMSO, and the final concentration of DMSO in the reaction mixture was $1 \%(\mathrm{v} / \mathrm{v})$. After preincubation at $37^{\circ} \mathrm{C}$ for 5 minutes, the reactions were initiated by the addition of cytosol samples. The reaction mixtures were incubated at $37^{\circ} \mathrm{C}$ for 15 (recombinant human SULTs), 30 (HIC), or 60 minutes (human liver, kidney, and lung cytosols), and the reaction was terminated by adding $100 \mu \mathrm{l}$ of ice-cold $4 \%$ (v/v) acetic acid acetonitrile containing F12994 as an internal standard. The protein concentration and reaction time for HLC and HIC assay were optimized based on linearity, in advance. To each terminated reaction mixture, $1 \mathrm{ml}$ of distilled water was added, and the sample was stirred. Standard curves were prepared as described previously herein, except that incubation was not included and sulfate conjugate standards were used instead of dotinurad.

Kinetic Analysis of Sulfation in HLC and HIC Samples. Kinetic studies were performed using pooled HLC and HIC samples. Sulfotransferase activities in presence of dotinurad concentrations ranging from 5 to $500 \mu \mathrm{M}$ were determined. The kinetic parameters were estimated in the same way as that for glucuronidation.

Inhibition Analysis of Sulfation in HLC and HIC Samples. Gavestinel and salbutamol were tested for their inhibitory effects on dotinurad sulfation in pooled HLC (only gavestinel used) and HIC samples. Gavestinel was used for the inhibition analysis of SULT1B1 (Senggunprai et al., 2009). Salbutamol was used for the inhibition analysis of SULT1A3 (Ko et al., 2012). Gavestinel was dissolved in DMSO, and salbutamol was dissolved in distilled water. Their concentrations in the reaction mixtures were adjusted to $10 \mu \mathrm{M}$ and $10 \mathrm{mM}$, respectively. Sulfotransferase activities were determined at $50 \mu \mathrm{M}$ dotinurad in a manner similar to that described previously herein

LC-MS/MS Analysis of Dotinurad Sulfate Conjugate. The sulfate conjugate in the reaction mixtures was quantified by LC-MS/MS using a Shimadzu Nexera HPLC system (Shimadzu Corporation, Kyoto, Japan) and QTRAP4500 (AB SCIEX). The terminated reaction mixtures were processed using solid-phase extraction in a 96-well plate format. The sulfate conjugate was eluted with 100 $\mu l$ of acetonitrile from the solid-phase and was diluted twice with distilled water. Then, $0.2 \mu 1$ of the processed reaction mixture was injected into an LC-MS/MS. Dotinurad sulfate conjugate and matrix constituents in reaction mixtures were separated using an Inertsil ODS-3 $(2.1 \times 150 \mathrm{~mm}, 3 \mu \mathrm{m}$; GL Sciences $)$ at $50^{\circ} \mathrm{C}$ with a mobile phase of $5 \mathrm{mM}$ ammonium acetate $(\mathrm{pH} 4)$ in water and methanol $(50: 50, \mathrm{v} / \mathrm{v})$. The total flow rate was set at $0.2 \mathrm{ml} / \mathrm{min}$. Ionization was conducted in turbo ion spray and negative ion modes. Dotinurad sulfate conjugate was analyzed as $[\mathrm{M}-\mathrm{H}]^{-}$ions in the multiple reaction monitoring mode (transitions: dotinurad sulfate conjugate 437.8/357.8 and internal standard F12994 340.9/ 145.0). For the reaction mixtures in the inhibition study, the mobile phase ratio was changed to wash the inhibitors, as described below. The initial mobile phase was $50 \% 5 \mathrm{mM}$ ammonium acetate $(\mathrm{pH} 4)$ in water and $50 \%$ methanol. The percentage of methanol was increased up to $95 \%$ at 12 minutes and maintained at 95\% at 12.1 to 25 minutes. From 25.1 to 35 minutes, the column was re-equilibrated with $50 \%$ methanol. The concentration range for the standard curve of sulfate conjugate was between 3 and $500 \mathrm{nM}$.

\section{Results}

Glucuronidation of Dotinurad by Human Tissue Microsomes. The glucuronosyltransferase activities for dotinurad in pooled human tissue microsomes (liver, intestine, and kidney) were determined. Figure 2 shows that HLMs exhibited a glucuronosyltransferase activity of 98.8 $\mathrm{pmol} / \mathrm{min}$ per milligram protein, which was more than 4-fold higher than that of human kidney and intestinal microsomes (23.6 and 12.6 $\mathrm{pmol} / \mathrm{min}$ per milligram protein, respectively). Kinetic analysis of dotinurad glucuronidation in HLMs and HKMs was performed. The glucuronidation in HLM followed the two-enzyme Michaelis-Menten kinetics, showing a biphasic Eadie-Hofstee plot, whereas the glucuronidation in HKM followed the Michaelis-Menten kinetics, showing a linear EadieHofstee plot (Fig. 3; Table 1). The apparent $\mathrm{K}_{\mathrm{m} \_\mathrm{HA}}$ and $\mathrm{K}_{\mathrm{m} \_ \text {LA }}$ of dotinurad glucuronidation in HLMs were 42.2 \pm 16.5 and 48,030 $\pm 820,500$ $\mu \mathrm{M}$ and the $\mathrm{V}_{\text {max_HA }}$ and $\mathrm{V}_{\text {max_LA }}$ were $166.5 \pm 29.2$ and $8564 \pm$ $144,000 \mathrm{pmol} / \mathrm{min}$ per milligram protein (mean \pm S.E.), respectively. The apparent $\mathrm{K}_{\mathrm{m}}$ and $\mathrm{V}_{\max }$ of dotinurad glucuronidation were $505.1 \pm$ $196.4 \mu \mathrm{M}$ and $263.7 \pm 62.4 \mathrm{pmol} / \mathrm{min}$ per milligram protein in HKMs, respectively. The $\mathrm{K}_{\mathrm{m}}$ of HKM was approximately 12-fold higher than the $K_{\mathrm{m} \_\mathrm{HA}}$ of HLM. With respect to the low-affinity component in HLM, $V_{\max }$ could not be calculated accurately due to the lack of highconcentration data.

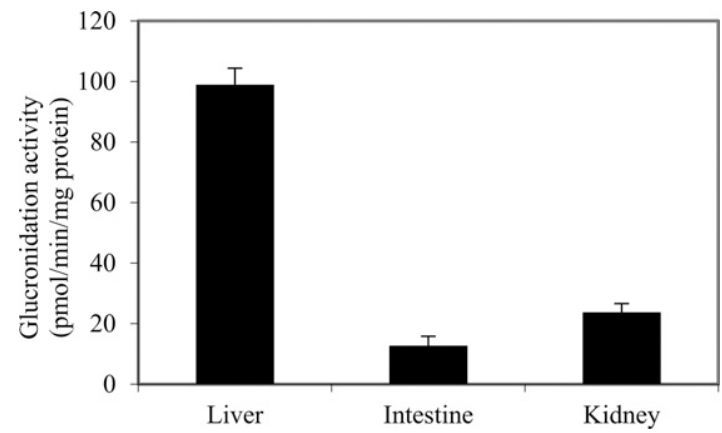

Fig. 2. Glucuronidation of dotinurad in pooled human tissue microsomes (liver, intestine, and kidney). Glucuronosyltransferase activities were determined as described in the Materials and Methods. Dotinurad concentration and microsome protein concentration were $50 \mu \mathrm{M}$ and $0.5 \mathrm{mg} / \mathrm{ml}$, respectively. Each column represents the mean \pm S.D. of triplicate determinations. 
A

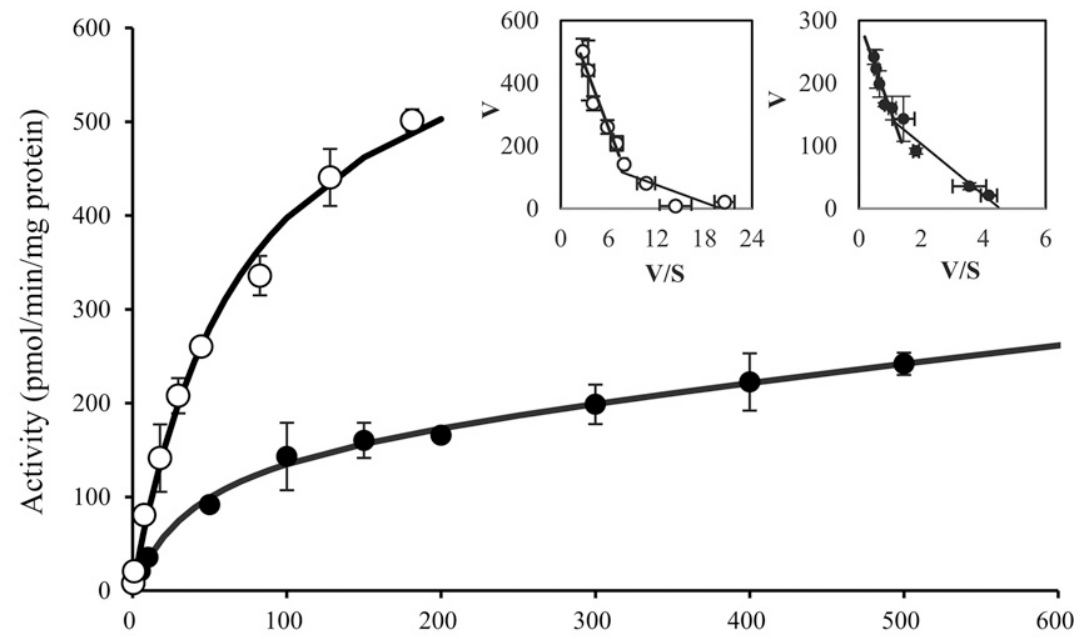

Substrate concentration $(\mu \mathrm{M})$

B

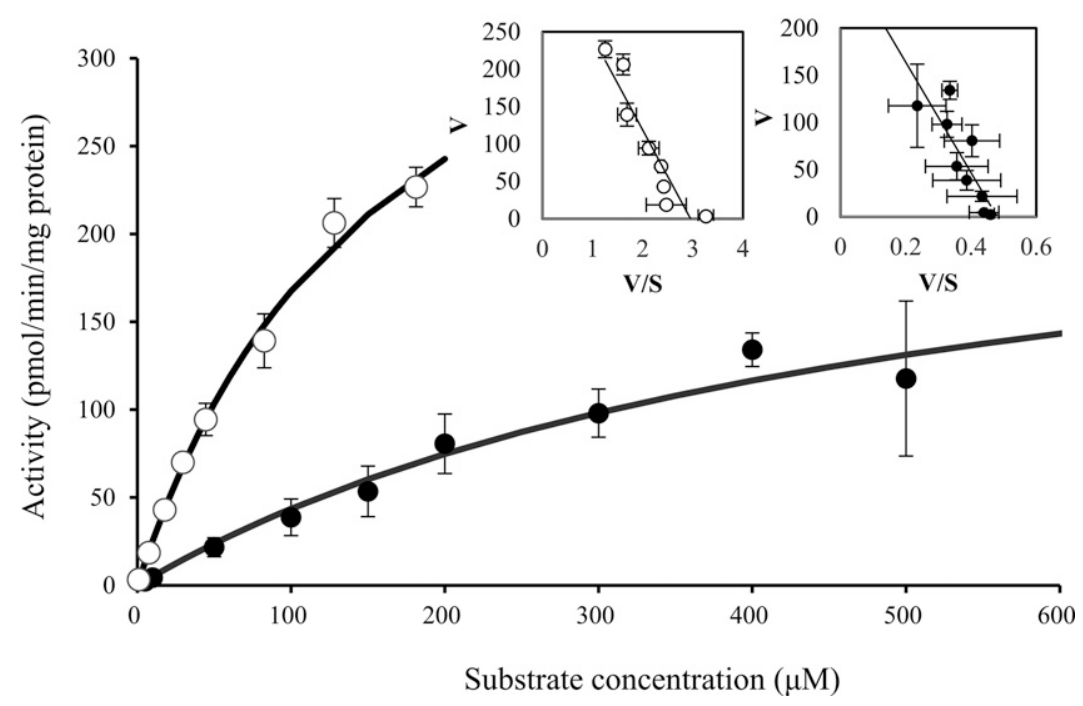

Fig. 3. Kinetics of glucuronidation in pooled HLMs (A) (with or without $1 \%$ BSA) and HKMs (B) (with or without $1 \%$ BSA). Glucuronosyltransferase activities were determined as described in the Materials and Methods. Each point represents the mean \pm S.D. of triplicate determinations. Solid circles, without $1 \%$ BSA; open circles, with $1 \%$ BSA. Each inset shows the Eadie-Hofstee plot of the experimental data. Dotinurad concentrations are unbound in the case of the reaction condition in the presence of $1 \% \mathrm{BSA}$.
Glucuronidation of Dotinurad by Recombinant Human UGT Isoforms. The glucuronosyltransferase activities of 13 recombinant UGT isoforms (UGT1A1, UGT1A3, UGT1A4, UGT1A6, UGT1A7, UGT1A8, UGT1A9, UGT1A10, UGT2B4, UGT2B7, UGT2B10, UGT2B15, and UGT2B17) for dotinurad were determined. Figure 4 shows that UGT1A3 exhibited a high glucuronosyltransferase activity (231.1 pmol/min per milligram protein) and that UGT1A1, 1A7, 1A8,
1A9, and 2B7 exhibited activities of 30.9, 8.4, 44.4, 25.6, and 21.9 $\mathrm{pmol} / \mathrm{min}$ per milligram protein, respectively. No other UGT isoforms showed glucuronosyltransferase activity (limit of quantification: 6.7 $\mathrm{pmol} / \mathrm{min}$ per milligram protein, calculated from quantitative value, reaction time, and protein concentration).

Inhibition Analysis of Glucuronidation in HLMs and HKMs. The effects of bilirubin, imipramine, diflunisal, and AZT on the

TABLE 1

Kinetic parameters of dotinurad glucuronidation using human tissue microsomes samples in the presence or absence of BSA Dotinurad was incubated with pooled HLM or HKM samples for 30 (HLMs) or 60 min (HKMs) without or with $1 \%$ BSA. The $K_{m}$ and $V_{m a x}$ values were estimated

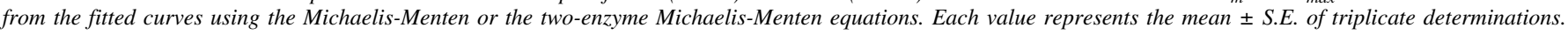

\begin{tabular}{|c|c|c|c|c|c|}
\hline Fraction & Condition & $\mathrm{K}_{\mathrm{m}}$ or $\mathrm{K}_{\mathrm{m} \_\mathrm{HA}}$ & $\mathrm{V}_{\max }$ or $\mathrm{V}_{\text {max_HA }}$ & $\mathrm{K}_{\mathrm{m} \_\mathrm{LA}}$ & $\mathrm{V}_{\max \_ \text {LA }}$ \\
\hline \multirow[t]{2}{*}{ HLMs } & $(-) 1 \%$ BSA & $\begin{array}{c}\mu M \\
42.2^{ \pm} \pm 16.5\end{array}$ & $\begin{array}{c}\text { pmol/min per milligram protein } \\
166.5 \pm 29.2\end{array}$ & $\begin{array}{c}\mu M \\
48,030 \pm 820,500\end{array}$ & $\begin{array}{l}\text { pmol/min per milligram protein } \\
8564 \pm 144,000\end{array}$ \\
\hline & $(+) 1 \% \mathrm{BSA}^{a}$ & $72.2 \pm 11.7$ & $684.3 \pm 48.7$ & - & - \\
\hline \multirow[t]{2}{*}{ HKMs } & $(-) 1 \%$ BSA & $505.1 \pm 196.4$ & $263.7 \pm 62.4$ & - & - \\
\hline & $(+) 1 \% \mathrm{BSA}^{a}$ & $162.8 \pm 21.0$ & $440.2 \pm 33.3$ & - & - \\
\hline
\end{tabular}

${ }^{a} \mathrm{~K}_{\mathrm{m}}$, unbound concentration in the case of the reaction condition in the presence of $1 \%$ BSA. 
Fig. 4. Screening of UGT isoforms for the glucuronide conjugate from dotinurad at a concentration of $10 \mu \mathrm{M}$. Each column represents the mean \pm S.D. of triplicate determinations. The lower limit of quantification of the assay under this condition was $6.7 \mathrm{pmol} / \mathrm{min}$ per milligram protein.

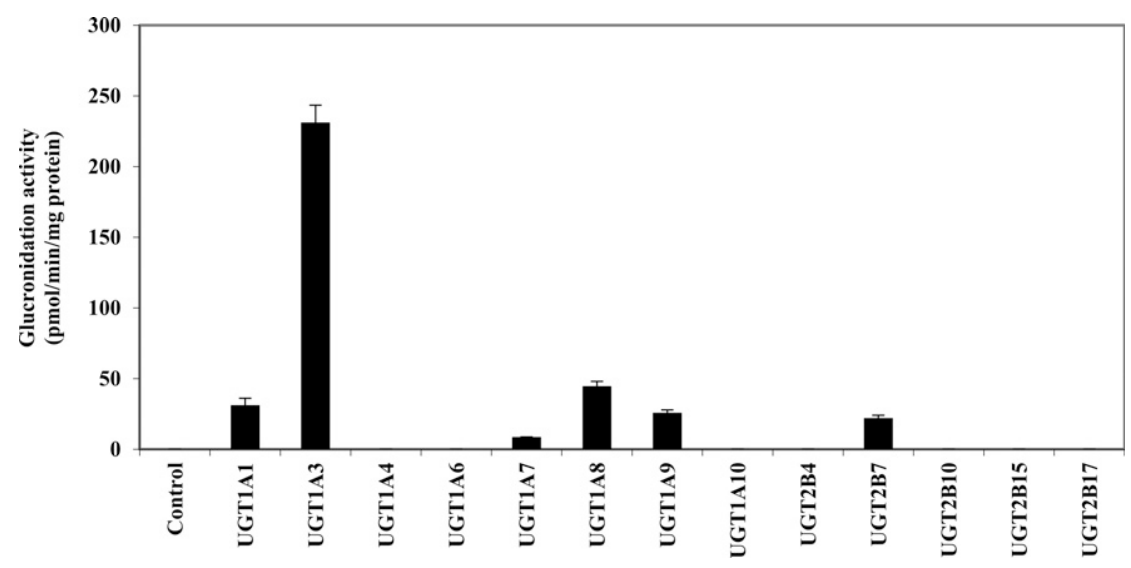

catalysis of dotinurad glucuronidation in HLMs and the effects of diflunisal and AZT on the catalysis of dotinurad glucuronidation in HKMs were tested. Bilirubin, imipramine, and diflunisal inhibited dotinurad glucuronidation in HLMs, and the percentages of inhibition were $45.5 \%$, $22.3 \%$, and $22.2 \%$ at 10,100 , and $50 \mu \mathrm{M}$, respectively (Fig. 5A). However, the inhibitory effects of AZT against dotinurad glucuronidation in HLMs were scarcely observed. The percentage of inhibition was $2.3 \%$ at $1 \mathrm{mM}$ (Fig. 5A). Diflunisal and AZT inhibited dotinurad glucuronidation in HKMs, and the percentages of inhibition were $61.1 \%$ and $17.5 \%$ at $50 \mu \mathrm{M}$ and $1000 \mu \mathrm{M}$, respectively (Fig. 5B).

Kinetic and Inhibition Analysis of Dotinurad Glucuronidation in HLMs and HKMs with 1\% BSA. Kinetic analysis of dotinurad glucuronidation in HLMs and HKMs with $1 \%$ BSA was conducted. The glucuronidation in HLM followed the Michaelis-Menten kinetics, showing a biphasic Eadie-Hofstee plot, whereas the glucuronidation in HKM followed the Michaelis-Menten kinetics, showing a linear Eadie-Hofstee plot (Fig. 3; Table 1). The dotinurad concentration used for the analysis was that unbound in the reaction mixture (Supplemental Table 1). The apparent $\mathrm{K}_{\mathrm{m}}$ and $\mathrm{V}_{\max }$ of dotinurad glucuronidation in HLMs were $72.2 \pm$ $11.7 \mu \mathrm{M}$ and $684.3 \pm 48.7 \mathrm{pmol} / \mathrm{min}$ per milligram protein (mean $\pm \mathrm{S}$. E.), respectively. The apparent $\mathrm{K}_{\mathrm{m}}$ and $\mathrm{V}_{\text {max }}$ of dotinurad glucuronidation were $162.8 \pm 21.0 \mu \mathrm{M}$ and $440.2 \pm 33.3 \mathrm{pmol} / \mathrm{min}$ per milligram protein in HKMs, respectively.

The effects of diflunisal and AZT on dotinurad glucuronidation in pooled HLMs and pooled HKMs with $1 \%$ BSA were also evaluated. Diflunisal and AZT inhibited dotinurad glucuronidation in HLMs, with the percentage of inhibition being $21.1 \%$ and $13.4 \%$ at 500 and 1200 $\mu \mathrm{M}$, respectively (Fig. 6A). Diflunisal and AZT inhibited dotinurad glucuronidation in HKMs, with the percentage of inhibition being $49.4 \%$ and $32.5 \%$ at 500 and $1200 \mu \mathrm{M}$, respectively (Fig. 6B). The concentrations of inhibitors were adjusted while considering protein binding in the reaction mixture. The unbound concentration of diflunisal in the reaction mixtures without $1 \%$ BSA was $38.5 \mu \mathrm{M}$ upon adding $50 \mu \mathrm{M}$ diflunisal, whereas that with $1 \%$ BSA was $46.8 \mu \mathrm{M}$ upon adding 500 $\mu \mathrm{M}$ diflunisal (Supplemental Table 2). AZT concentration was selected based on the unbound fraction rate reported by Kilford et al. (2009): 0.6 in the absence of $2 \%$ BSA and 0.49 in the presence of $2 \%$ BSA.

Sulfation of Dotinurad by Human Tissue Cytosol Samples. The sulfotransferase activities for dotinurad in pooled human tissue cytosol samples (liver, intestine, kidney, and lung) were determined. Figure 7 shows that HIC samples exhibited a sulfotransferase activity of $29.0 \mathrm{pmol} / \mathrm{min}$ per milligram protein, which was more than 3.9-fold higher than that of human liver, kidney, and lung cytosol samples (7.5,
0.5 , and $1.5 \mathrm{pmol} / \mathrm{min}$ per milligram protein, respectively). Kinetic analysis of dotinurad sulfation in HLC and HIC samples was performed. In HLC, the sulfation followed the two-enzyme Michaelis-Menten kinetics, showing a biphasic Eadie-Hofstee plot, whereas in HIC, the sulfation followed the Michaelis-Menten kinetics, showing a biphasic EadieHofstee plot (Fig. 8; Table 2). The apparent $\mathrm{K}_{\mathrm{m} \_\mathrm{HA}}$ and $\mathrm{K}_{\mathrm{m} \_\mathrm{LA}}$ of
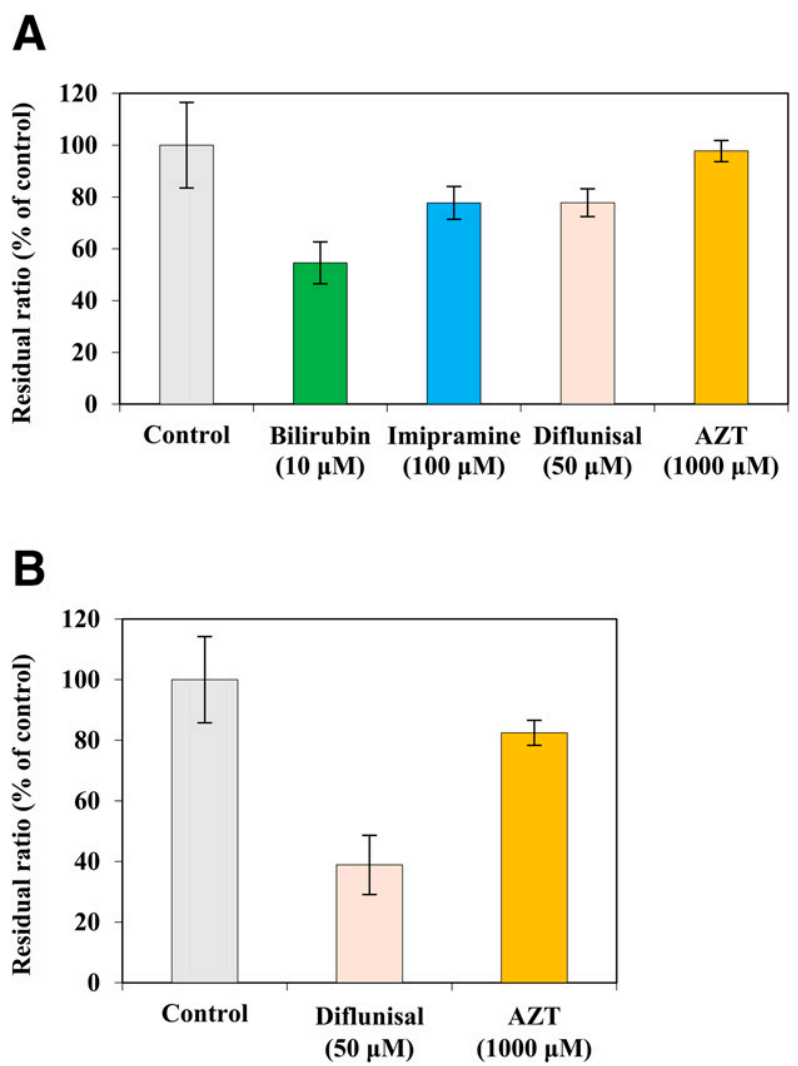

Fig. 5. Effects of bilirubin, imipramine, diflunisal, and AZT on dotinurad glucuronidation in pooled HLMs (A) and effects of diflunisal and AZT on dotinurad glucuronidation in pooled HKMs (B). The glucuronosyltransferase activities for dotinurad were measured at a concentration of $10 \mu \mathrm{M}$ under coincubation with either $10 \mu \mathrm{M}$ bilirubin, $100 \mu \mathrm{M}$ imipramine, $50 \mu \mathrm{M}$ diflunisal, or $1000 \mu \mathrm{M}$ AZT. The control activities for dotinurad glucuronidation in the pooled HLMs and HKMs in the absence of inhibitors were 90.9 and $23.9 \mathrm{pmol} / \mathrm{min}$ per milligram protein, respectively. Each column represents the mean \pm S.D. of triplicate determinations. 
A

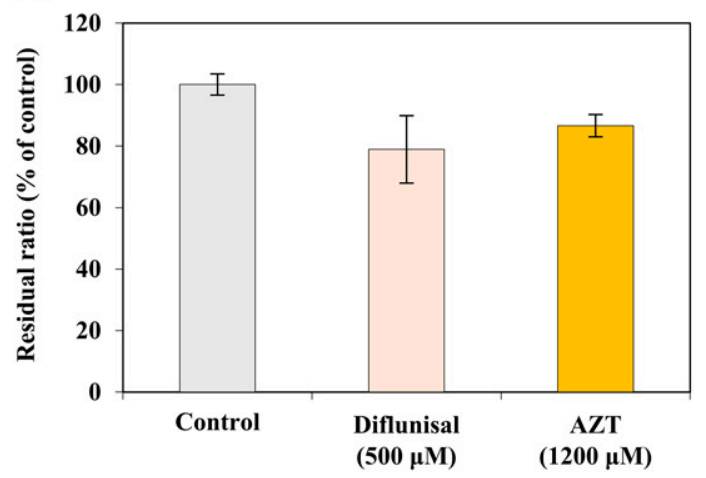

B

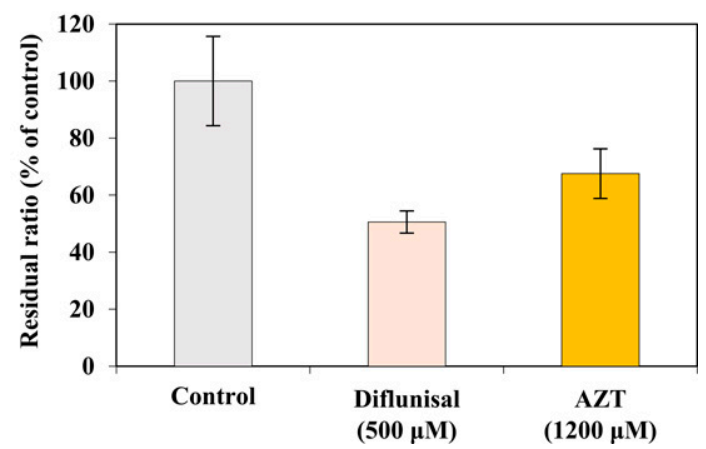

Fig. 6. Effects of diflunisal and AZT on dotinurad glucuronidation in pooled human liver microsomes with 1\% BSA [HLMs (A)] and pooled human kidney microsomes with $1 \%$ BSA [HKMs (B)]. The glucuronosyltransferase activities for dotinurad were measured at a total concentration of $50 \mu \mathrm{M}$ (HLMs) or 150 $\mu \mathrm{M}$ (HKMs) under coincubation with either $500 \mu \mathrm{M}$ diflunisal or $1200 \mu \mathrm{M}$ AZT. The control activities for dotinurad glucuronidation in the pooled HLMs and pooled HKMs with $1 \%$ BSA in the absence of inhibitors were 59.8 and 52.9 $\mathrm{pmol} / \mathrm{min}$ per milligram protein, respectively. Each column represents the mean \pm S.D. of triplicate determinations.

dotinurad sulfation in HLCs were $8.4 \pm 3.4$ and $279.4 \pm 111.4 \mu \mathrm{M}$, and the $\mathrm{V}_{\text {max_HA }}$ and $\mathrm{V}_{\text {max_LA }}$ were $6.3 \pm 1.5$ and $16.3 \pm 1.1 \mathrm{pmol} / \mathrm{min}$ per milligram protein (mean \pm S.E.), respectively. The apparent $K_{m}$ and $\mathrm{V}_{\max }$ of dotinurad sulfation in HICs were $305.6 \pm 128.8 \mu \mathrm{M}$ and $124.7 \pm 26.8 \mathrm{pmol} / \mathrm{min}$ per milligram protein, respectively.

Sulfation of Dotinurad by Recombinant Human SULT Isoforms. The sulfotransferase activities of eight recombinant SULT isoforms (SULT1A1*1, SULT1A2, SULT1A3, SULT1B1, SULT1C2,

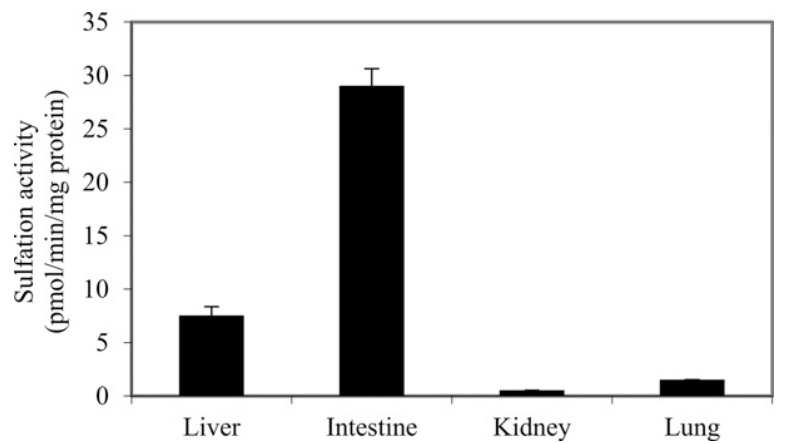

Fig. 7. Sulfation of dotinurad in pooled human tissue cytosol samples (liver, intestine, kidney, and lung). Sulfotransferase activities were determined as described in the Materials and Methods. Dotinurad concentration and cytosol protein concentration were $50 \mu \mathrm{M}$ and 0.1 (intestine only) or $0.5 \mathrm{mg} / \mathrm{ml}$, respectively. Each column represents the mean \pm S.D. of triplicate determinations.
A

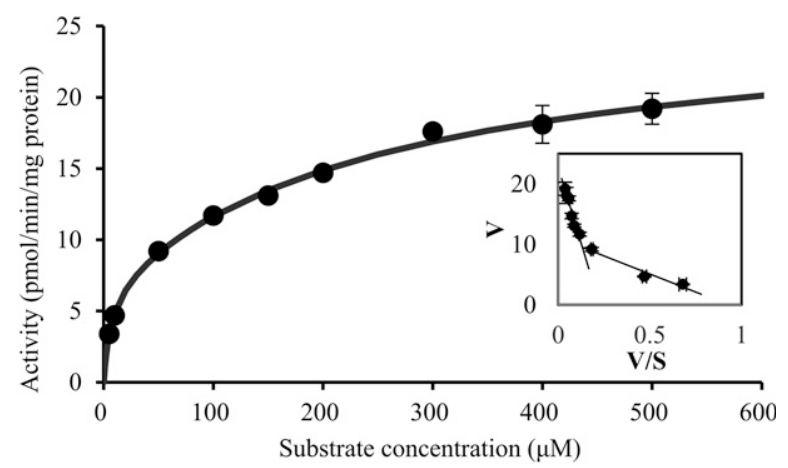

B

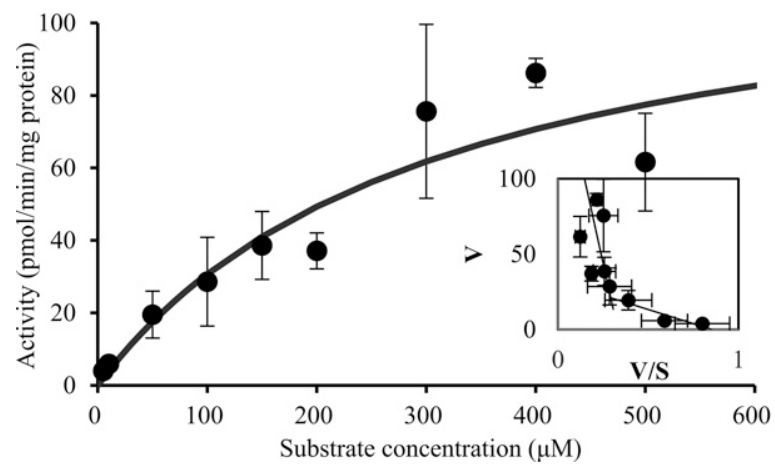

Fig. 8. Kinetics of sulfation in pooled human liver cytosol [HLC (A)] and human intestine cytosol [HIC (B)] samples. Sulfotransferase activities were determined as described in the Materials and Methods. Each point represents the mean \pm S.D. of triplicate determinations, and each inset shows the Eadie-Hofstee plot of the experimental data.

SULT1C4, SULT1E1, and SULT2A1) for dotinurad were determined. Figure 9 shows that SULT1A3, SULT1B1, SULT1C4, and SULT1E1 exhibited relatively high sulfotransferase activities of 658.7, 438.5, 473.3, and $1074.7 \mathrm{pmol} / \mathrm{min}$ per milligram protein, respectively. Although the sulfotransferase activities of other SULTs were low (SULT1A1*1, SULT1A2, SULT1C2, and SULT2A1: 111.3, 90.0, 88.2, and 110.3 $\mathrm{pmol} / \mathrm{min}$ per milligram protein, respectively), all SULT isoforms used in this study catalyzed the formation of the sulfate conjugate.

Inhibition Analysis of Sulfation in HLC and HIC Samples. The effects of gavestinel and salbutamol on the catalysis of dotinurad sulfation in pooled HLC and pooled HIC samples were tested. The percentages of inhibition of gavestinel for dotinurad sulfation in HLCs and HICs were $15.3 \%$ and $6.6 \%$, respectively, at a concentration of $10 \mu \mathrm{M}$ (Fig. 10, A and B). Salbutamol inhibited dotinurad sulfation in HICs, with a percent inhibition of $68.4 \%$ at $10 \mathrm{mM}$ (Fig. 10B).

\section{Discussion}

The present study aimed to identify the human UGT and SULT isoforms responsible for dotinurad glucuronidation and sulfation. First, dotinurad glucuronidation by HLMs, HIMs, and HKMs was examined (Fig. 2). The glucuronidation activities of HLMs, HIMs, and HKMs against dotinurad, corrected by the microsome content of the liver, intestine, and kidney ( $40.0 \mathrm{mg} / \mathrm{g}$ liver, $20.6 \mathrm{mg} / \mathrm{g}$ intestine mucosal scrapings, and $11.1 \mathrm{mg} / \mathrm{g}$ kidney), as well as organ weights $(21.4 \mathrm{~g}$ liver $/ \mathrm{kg}, 1.35 \mathrm{~g}$ intestine mucosal scrapings $/ \mathrm{kg}$, and $4.5 \mathrm{~g}$ kidney $/ \mathrm{kg}$ ) (Gibbs et al., 1998; Cubitt et al., 2011; Scotcher et al., 2017), were $84.6,0.3$, and $1.1 \mathrm{nmol} / \mathrm{min}$ per kilogram, respectively, suggesting that 
TABLE 2

Kinetic parameters of dotinurad sulfation using HLCs or HICs

Dotinurad was incubated with HLCs or HICs samples for 30 (HICs) or 60 min (HLCs). The $K_{m}$ and $V_{\text {max }}$ values were estimated from the fitted curves using the Michaelis-Menten or the two-enzyme Michaelis-Menten equation. Each value represents the mean \pm S.E. of triplicate determinations.

\begin{tabular}{|c|c|c|c|c|}
\hline Fraction & $\mathrm{K}_{\mathrm{m}}$ or $\mathrm{K}_{\mathrm{m} \_\mathrm{HA}}$ & $\mathrm{V}_{\max }$ or $\mathrm{V}_{\text {max_HA }}$ & $\mathrm{K}_{\mathrm{m} \_\mathrm{LA}}$ & $\mathrm{V}_{\max \_L A}$ \\
\hline & $\mu M$ & pmol/min per milligram protein & $\mu M$ & pmol/min per milligram protein \\
\hline HLCs & $8.4 \pm 3.4$ & $6.3 \pm 1.5$ & $279.4 \pm 111.4$ & $16.3 \pm 1.1$ \\
\hline $\mathrm{HICs}$ & $305.6 \pm 128.8$ & $124.7 \pm 26.8$ & - & - \\
\hline
\end{tabular}

dotinurad glucuronide conjugate was generated primarily in the liver. Kinetic analysis revealed that the Eadie-Hofstee plot of dotinurad glucuronidation in HLMs was biphasic (Fig. 3A), indicating that multiple UGTs were involved in dotinurad glucuronidation in HLMs. Next, dotinurad glucuronidation in the presence of 13 commercially available recombinant human UGTs was examined. Among them, dotinurad glucuronidation activities of UGT1A1, UGT1A3, UGT1A7, UGT1A8, UGT1A9, and UGT2B7 were observed (Fig. 4). UGT1A1, UGT1A3, UGT1A9, and UGT2B7 are expressed in the liver. The protein levels of these isoforms in HLMs were 124, 20.6, 61.1, and $200 \mathrm{pmol} / \mathrm{mg}$ protein, respectively. However, UGT1A7 and UGT1A8 are not expressed in the liver (Sato et al., 2014). Therefore, as the UGT isoforms involved in dotinurad glucuronidation are thought to be UGT1A1, UGT1A3, UGT1A9, and UGT2B7, the contribution of each UGT isoform to dotinurad glucuronidation was investigated via an inhibition study (Fig. 5). The percentages of inhibition in HLMs were 45.5\%, 22.3\%, 22.2\%, and $2.3 \%$, relative to control activity, when bilirubin, imipramine, diflunisal, and AZT were added, respectively. In the preliminary study, we determined whether each inhibitor was selective against UGT1A1, UGT1A3, UGT1A9, or UGT2B7. Treatment with $100 \mu \mathrm{M}$ bilirubin resulted in inhibition overestimation as it inhibited dotinurad glucuronidation by other UGTs besides UGT1A1 by approximately $50 \%$. Therefore, although the percent inhibition by $10 \mu \mathrm{M}$ bilirubin (45.5\%) did not accurately demonstrate the contribution of UGT1A1 to dotinurad glucuronidation due to weak inhibition, we considered UGT1A1 to be involved in dotinurad glucuronidation. With respect to the other inhibitors, imipramine inhibited UGT1A1 (percent inhibition; 25.8\%), UGT1A3 (71.6\%), UGT1A9 (0.0\%), and UGT2B7 (47.3\%); diflunisal inhibited UGT1A1 (29.0\%), UGT1A3 (9.3\%), UGT1A9 (94.0\%), and UGT2B7 (20.4\%); and AZT inhibited UGT1A1 (-2.2\%), UGT1A3 (-5.5\%), UGT1A9 (13.7\%), and UGT2B7 (76.7\%). The glucuronidation activities of UGT1A9 and UGT2B7 might have been underestimated due to inhibition by fatty acid in microsomes. Therefore, we conducted a study in which $1 \%$ BSA was added to the reaction mixtures for trapping fatty acids. The $\mathrm{K}_{\mathrm{m}}$ of dotinurad glucuronidation in HKMs with $1 \%$ BSA was lower than that of HKMs without $1 \%$ BSA, whereas the $\mathrm{V}_{\max }$ in HKMs with $1 \%$ BSA was higher than that without $1 \%$ BSA (Table 1). Furthermore, although dotinurad glucuronidation in HLMs with $1 \%$ BSA did not fit the two-enzyme Michaelis-Menten equation well, it is possible that UGT1A9 and UGT2B7 are low-affinity components in HLMs, considering the $\mathrm{K}_{\mathrm{m}}$ of HKMs. Additionally, AZT inhibited dotinurad glucuronidation in HLMs and HKMs with $1 \%$ BSA more strongly than it did without $1 \%$ BSA (Figs. 5 and 6). The contribution of UGT2B7 to dotinurad glucuronidation was attributed to $1 \%$ BSA. These results suggest that dotinurad glucuronidation is catalyzed primarily by UGT1A1, UGT1A3, UGT1A9, and UGT2B7.

Dotinurad sulfation by human liver, intestine, kidney, and lung cytosol samples was also examined (Fig. 7). The sulfation activities of HLCs and HICs for dotinurad were high. These activities were then corrected by the cytosol content of liver and small intestine $(80.7 \mathrm{mg} / \mathrm{g}$ liver and $18.0 \mathrm{mg} / \mathrm{g}$ intestine mucosal scrapings) and organ weight (21.4 g liver/kg and $1.35 \mathrm{~g}$ intestine mucosal scrapings $/ \mathrm{kg}$ ) (Gibbs et al., 1998; Cubitt et al., 2011), yielding values of 13.0 and $0.7 \mathrm{nmol} / \mathrm{min}$ per kilogram, respectively, suggesting that dotinurad sulfate conjugate was generated primarily in the liver. Kinetic analysis revealed that the Eadie-Hofstee plots of dotinurad sulfation in HLCs and HICs were biphasic (Fig. 8, A and B), indicating that several SULTs were involved in dotinurad sulfation. Next, dotinurad sulfation by eight commercially available recombinant human SULTs was examined (Fig. 9). The sulfation activities of all SULT isoforms for dotinurad were observed. Among them, SULT1A1, SULT1A3, SULT1B1, SULT1E1, and SULT2A1 are important SULT isoforms, and their expression levels in humans have been reported (Riches et al., 2009). SULT1A1 and SULT2A1 may contribute to dotinurad sulfation, considering the
Fig. 9. Screening of SULT isoforms for the sulfate conjugate from dotinurad at a concentration of $10 \mu \mathrm{M}$. Each column represents the mean \pm S.D. of triplicate determinations. The lower limit of quantification of the assay under this condition was 2 (1C2 and 2A1), 4 (1A1 and 1B1), and $20(1 \mathrm{~A} 2,1 \mathrm{~A} 3,1 \mathrm{C} 4$, and $1 \mathrm{E} 1) \mathrm{pmol} / \mathrm{min}$ per milligram protein.

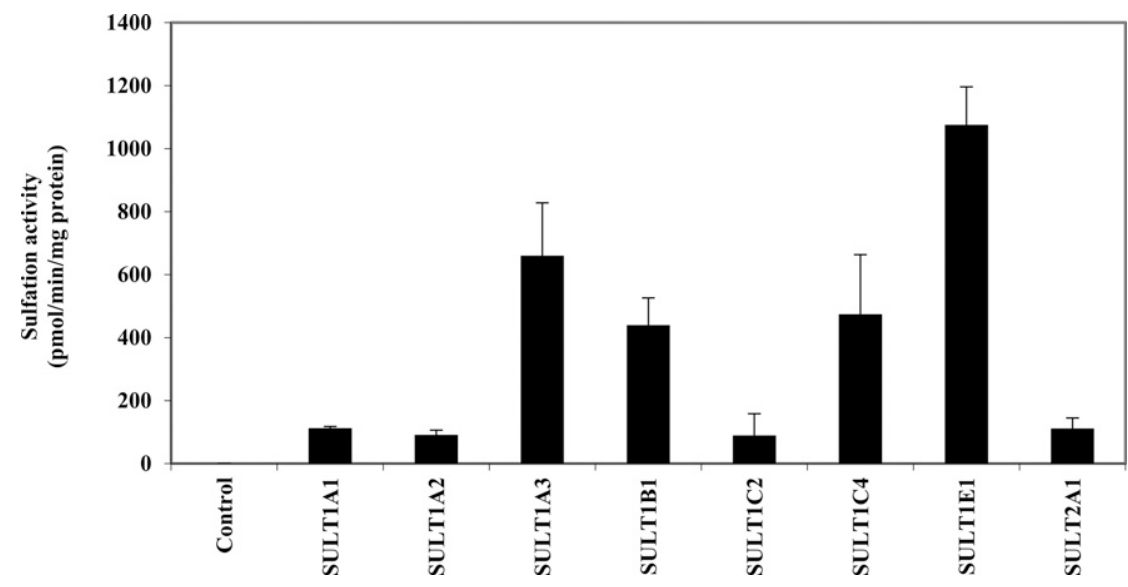



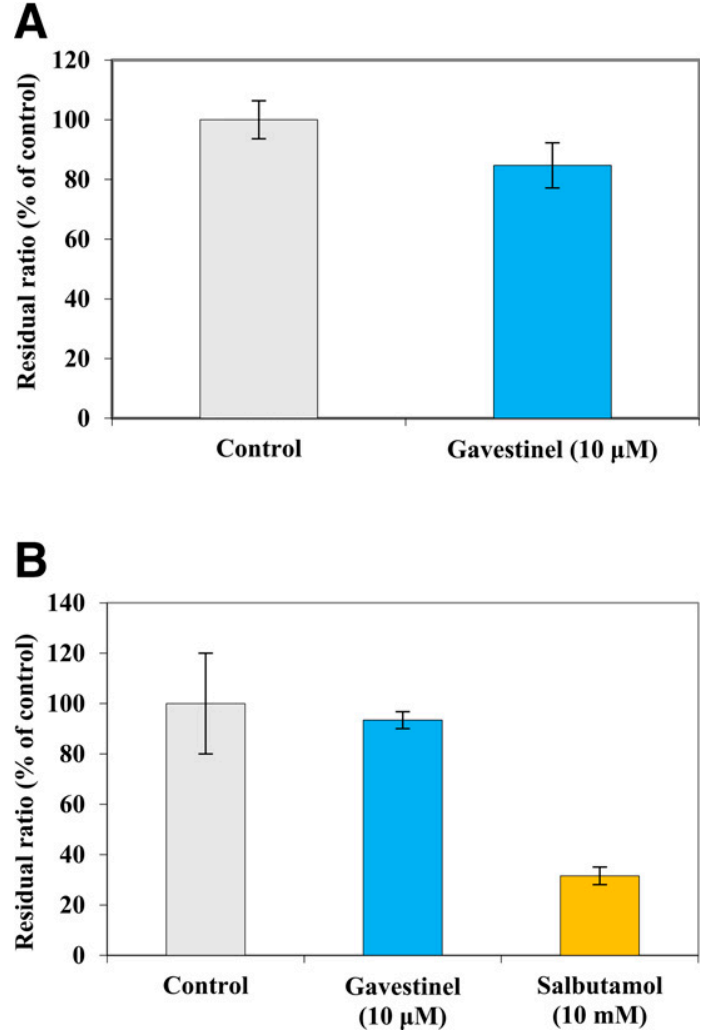

Fig. 10. Effects of gavestinel on dotinurad sulfation in pooled human liver cytosol [HLC (A)] samples and effects of gavestinel and salbutamol on dotinurad sulfation in pooled human intestine cytosol [HIC (B)] samples. The sulfotransferase activities for dotinurad were measured at a concentration of $50 \mu \mathrm{M}$ under coincubation with either $10 \mu \mathrm{M}$ gavestinel or $10 \mathrm{mM}$ salbutamol. The control activities for dotinurad sulfation in the pooled HLCs and HICs in the absence of inhibitors were 8.5 and $25.3 \mathrm{pmol} / \mathrm{min}$ per milligram protein, respectively. Each column represents the mean \pm S.D. of triplicate determinations.

amount of expression in the human liver. Additionally, an inhibition study was conducted using selective and potent inhibitors observed during the preliminary study [gavestinel inhibited SULT1A1 (19.6\%), SULT1A3 (2.3\%), SULT1B1 (98.1\%), SULT1E1 (20.9\%), and SULT2A1 (21.9\%), and salbutamol inhibited SULT1A1 (11.7\%), SULT1A3 (84.8\%), SULT1B1 $(-12.4 \%)$, SULT1E1 $(-7.0 \%)$, and SULT2A1 $(-7.8 \%)]$. The percent inhibition in HLCs was $15.3 \%$, relative to control activity, when gavestinel, a SULT1B1 inhibitor, was added (Fig. 10A). These results suggest that dotinurad sulfation is catalyzed by more than one SULT isoform, although the contribution of each SULT isoform is unclear. The contribution of SULT1A3 was low, possibly as a result of the lack of its expression in the human liver. However, salbutamol strongly inhibited dotinurad sulfation in HIC samples; therefore, SULT1A3 may play an important role in dotinurad sulfation in the gastrointestinal tract when dotinurad is absorbed. However, the intestinal availability of dotinurad is considered high, as the $\mathrm{V}_{\max } / \mathrm{K}_{\mathrm{m}}$ of dotinurad in HICs is very low $(0.41 \mu \mathrm{l} / \mathrm{min}$ per milligram protein $)$ in comparison with the $\mathrm{V}_{\max } / \mathrm{K}_{\mathrm{m}}$ of salbutamol (intestinal availability $=0.7$; Mizuma et al., 2005) in SULT1A3 (230 $\mu \mathrm{l} / \mathrm{min}$ per milligram protein) (Ko. et al., 2012). Moreover, the bioavailability of dotinurad is also considered high because of the very low oral clearance of dotinurad (0.013 1/h per kilogram; Omura et al., 2020).

For the safe usage of a drug, it is helpful to predict DDIs and the interindividual variability in its metabolic activity. Dotinurad is mainly eliminated through metabolic clearance, and $44.3 \%$ of the dose is excreted via the urine as a glucuronide conjugate. Accordingly, we evaluated drug interactions based on the inhibition of dotinurad glucuronidation in HLMs using 21 drugs that are expected to be used concomitantly with dotinurad in clinical situations. The result indicated that oxaprozin was the most potent inhibitor of dotinurad glucuronidation (Supplemental Table 3). However, the ratios of the area under the concentration-time curve from time 0 to infinity and oral clearance after coadministration with oxaprozin compared with those with the administration of dotinurad alone were 1.165 and 0.858 , respectively, in the clinical DDI study (Furihata et al., 2020). The risk of DDIs caused by concomitant drugs that inhibit UGTs is assumed to be low, as there are multiple UGTs involved in dotinurad glucuronidation, and hence it is difficult for concomitant drugs to inhibit all UGT isoforms.

Several single nucleotide polymorphisms, which are one of the causes of interindividual differences, have been identified in the UGT and SULT that are involved in dotinurad metabolism. For example, it has been reported that the SN-38 glucuronidation activity of UGT1A1*28 [(TA)7TAA, instead of (TA)6TAA], is lower than that of wild-type UGT1A1 (Iyer et al., 2002) and that examining the enzyme activities of SULT1A1*1, *2, and *3 with various substrates showed that the $\mathrm{V}_{\text {max }}$ was $* 1>* 3>* 2$, whereas the $\mathrm{K}_{\mathrm{m}}$ varied based on the substrate (Nagar et al., 2006). The differences in the activity of the respective single nucleotide polymorphisms are large; however, the overall change in the activity of enzymes that catalyze dotinurad glucuronidation and sulfation is suppressed because multiple UGT and SULT isoforms are involved in dotinurad metabolism.

It has been reported that body fat area affects serum uric acid levels (Takahashi et al., 1997; Matsuura et al., 1998), and therefore, it is highly possible that patients with hyperuricemia with liver diseases, such as nonalcoholic steatohepatitis or steatosis, will receive dotinurad. Moreover, UGT and SULT expression in liver disease has been reported. Congiu et al. (2002) reported that interindividual variation for UGT2B17 was the greatest, whereas the expression of UGT2B7 was reduced to $38.4 \%$ to that of the control level in biopsies from patients with high inflammation scores. Furthermore, it has been reported that UGT1A9, SULT1A1, and SULT2A1 protein levels are decreased in nonalcoholic steatohepatitis (Hardwick et al., 2013), possibly because of the decrease in the activities of certain UGT and SULT isoforms during liver disease. However, the change in dotinurad metabolic clearance remains small as the activities of several UGT and SULT isoforms are maintained. Indeed, no significant differences in the pharmacokinetic parameters of dotinurad were observed between subjects with hepatic impairment and those with normal hepatic function (Kumagai et al., 2020).

We do not consider DDI and the variability of metabolic activity to cause limitation since dotinurad has a wide margin of safety, while several other metabolic enzymes contribute to its metabolism. However, if more information was available on selective inhibitors of UGT and SULT, the predictability of DDIs or interindividual differences would improve. In conclusion, dotinurad is a selective urate reabsorption inhibitor that can be safely used because of the small risk of DDIs and low interindividual variability caused by the involvement of many UGT and SULT isoforms in its metabolism.

\section{Acknowledgments}

We appreciate Masato Inaba, Research Institute, Fuji Yakuhin Co., Ltd., for supplying glucuronide conjugate and sulfate conjugate of dotinurad. We would like to thank Editage (www.editage.com) for English language editing.

\section{Authorship Contributions}

Participated in research design: Omura, Miyata, Yamano, Iwanaga. Conducted experiments: Omura. 
Contributed new reagents or analytic tools: Kobashi.

Performed data analysis: Omura, Motoki.

Wrote or contributed to the writing of the manuscript: Omura, Yamano, Iwanaga.

\section{References}

Congiu M, Mashford ML, Slavin JL, and Desmond PV (2002) UDP glucuronosyltransferase mRNA levels in human liver disease. Drug Metab Dispos 30:129-134.

Court MH, Krishnaswamy S, Hao Q, Duan SX, Patten CJ, Von Moltke LL, and Greenblatt DJ (2003) Evaluation of $3^{\prime}$-azido-3'-deoxythymidine, morphine, and codeine as probe substrates for UDP-glucuronosyltransferase 2B7 (UGT2B7) in human liver microsomes: specificity and influence of the UGT2B7*2 polymorphism. Drug Metab Dispos 31:1125-1133.

Cubitt HE, Houston JB, and Galetin A (2011) Prediction of human drug clearance by multiple metabolic pathways: integration of hepatic and intestinal microsomal and cytosolic data. Drug Metab Dispos 39:864-873.

Furihata K, Nagasawa K, Hagino A, and Kumagai Y (2020) A drug-drug interaction study of a novel, selective urate reabsorption inhibitor dotinurad and the non-steroidal anti-inflammatory drug oxaprozin in healthy adult males. Clin Exp Nephrol 24 (Suppl 1):36-43.

Gibbs JP, Yang JS, and Slattery JT (1998) Comparison of human liver and small intestinal glutathione S-transferase-catalyzed busulfan conjugation in vitro. Drug Metab Dispos 26:52-55.

Hardwick RN, Ferreira DW, More VR, Lake AD, Lu Z, Manautou JE, Slitt AL, and Cherrington NJ (2013) Altered UDP-glucuronosyltransferase and sulfotransferase expression and function during progressive stages of human nonalcoholic fatty liver disease. Drug Metab Dispos 41:554-561.

Iyer L, Das S, Janisch L, Wen M, Ramírez J, Karrison T, Fleming GF, Vokes EE, Schilsky RL, and Ratain MJ (2002) UGT1A1*28 polymorphism as a determinant of irinotecan disposition and toxicity. Pharmacogenomics $J$ 2:43-47.

Kamali F (1993) The effect of probenecid on paracetamol metabolism and pharmacokinetics. Eur J Clin Pharmacol 45:551-553.

Kilford PJ, Stringer R, Sohal B, Houston JB, and Galetin A (2009) Prediction of drug clearance by glucuronidation from in vitro data: use of combined cytochrome P450 and UDP-glucuronosyltransferase cofactors in alamethicin-activated human liver microsomes. Drug Metab Dispos 37:82-89.

Ko K, Kurogi K, Davidson G, Liu MY, Sakakibara Y, Suiko M, and Liu MC (2012) Sulfation of ractopamine and salbutamol by the human cytosolic sulfotransferases. J Biochem 152:275-283.

Kumagai Y, Sakaki M, Furihata K, Ito T, Inoue K, Yoshida T, Matsumoto S, Furuno K, and Hagino A (2020) Dotinurad: a clinical pharmacokinetic study of a novel, selective urate reabsorption inhibitor in subjects with hepatic impairment. Clin Exp Nephrol 24 (Suppl 1):25-35.

Matsuura F, Yamashita S, Nakamura T, Nishida M, Nozaki S, Funahashi T, and Matsuzawa Y (1998) Effect of visceral fat accumulation on uric acid metabolism in male obese subjects: visceral fat obesity is linked more closely to overproduction of uric acid than subcutaneous fat obesity. Metabolism 47:929-933.

Meech R, Hu DG, McKinnon RA, Mubarokah SN, Haines AZ, Nair PC, Rowland A, and Mackenzie PI (2019) The UDP-glycosyltransferase (UGT) superfamily: new members, new functions, and novel paradigms. Physiol Rev 99:1153-1222.
Mizuma T, Kawashima K, Sakai S, Sakaguchi S, and Hayashi M (2005) Differentiation of organ availability by sequential and simultaneous analyses: intestinal conjugative metabolism impacts on intestinal availability in humans. J Pharm Sci 94:571-575.

Nagar S, Walther S, and Blanchard RL (2006) Sulfotransferase (SULT) 1A1 polymorphic variants $* 1, * 2$, and $* 3$ are associated with altered enzymatic activity, cellular phenotype, and protein degradation. Mol Pharmacol 69:2084-2092.

Omura K, Miyata K, Kobashi S, Ito A, Fushimi M, Uda J, Sasaki T, Iwanaga T, and Ohashi T (2020) Ideal pharmacokinetic profile of dotinurad as a selective urate reabsorption inhibitor. Drug Metab Pharmacokinet 35:313-320.

Riches Z, Stanley EL, Bloomer JC, and Coughtrie MW (2009) Quantitative evaluation of the expression and activity of five major sulfotransferases (SULTs) in human tissues: the SULT "pie". Drug Metab Dispos 37:2255-2261.

Sato Y, Nagata M, Tetsuka K, Tamura K, Miyashita A, Kawamura A, and Usui T (2014) Optimized methods for targeted peptide-based quantification of human uridine $5^{\prime}$-diphosphate-glucuronosyltransferases in biological specimens using liquid chromatography-tandem mass spectrometry. Drug Metab Dispos 42:885-889.

Scotcher D, Billington S, Brown J, Jones CR, Brown CDA, Rostami-Hodjegan A, and Galetin A (2017) Microsomal and cytosolic scaling factors in dog and human kidney cortex and application for in vitro-in vivo extrapolation of renal metabolic clearance. Drug Metab Dispos 45:556-568.

Senggunprai L, Yoshinari K, and Yamazoe Y (2009) Inhibitory effects of kynurenic acid, a tryptophan metabolite, and its derivatives on cytosolic sulfotransferases. Biochem J 422:455-462.

Shiraga T, Yajima K, Suzuki K, Suzuki K, Hashimoto T, Iwatsubo T, Miyashita A, and Usui T (2012) Identification of UDP-glucuronosyltransferases responsible for the glucuronidation of darexaban, an oral factor Xa inhibitor, in human liver and intestine. Drug Metab Dispos 40:276-282.

Suiko M, Kurogi K, Hashiguchi T, Sakakibara Y, and Liu MC (2017) Updated perspectives on the cytosolic sulfotransferases (SULTs) and SULT-mediated sulfation. Biosci Biotechnol Biochem 81:63-72.

Takahashi S, Yamamoto T, Tsutsumi Z, Moriwaki Y, Yamakita J, and Higashino K (1997) Close correlation between visceral fat accumulation and uric acid metabolism in healthy men. Metabolism 46:1162-1165.

Takano M and Sugiyama T (2017) UGT1A1 polymorphisms in cancer: impact on irinotecan treatment. Pharm Genomics Pers Med 10:61-68.

Taniguchi T, Ashizawa N, Matsumoto K, Saito R, Motoki K, Sakai M, Chikamatsu N, Hagihara C, Hashiba M, and Iwanaga T (2019) Pharmacological evaluation of dotinurad, a selective urate reabsorption inhibitor. J Pharmacol Exp Ther 371:162-170.

Uda J, Kobashi S, Miyata S, Ashizawa N, Matsumoto K, and Iwanaga T (2020) Discovery of dotinurad (FYU-981), a new phenol derivative with highly potent uric acid lowering activity. ACS Med Chem Lett 11:2017-2023.

Walsky RL, Bauman JN, Bourcier K, Giddens G, Lapham K, Negahban A, Ryder TF, Obach RS, Hyland R, and Goosen TC (2012) Optimized assays for human UDP-glucuronosyltransferase (UGT) activities: altered alamethicin concentration and utility to screen for UGT inhibitors. Drug Metab Dispos 40:1051-1065.

Yamanaka H, Nakajima M, Katoh M, and Yokoi T (2007) Glucuronidation of thyroxine in human liver, jejunum, and kidney microsomes. Drug Metab Dispos 35:1642-1648.

Yasuda K, Ikushiro S, Kamakura M, Munetsuna E, Ohta M, and Sakaki T (2011) Sequential metabolism of sesamin by cytochrome P450 and UDP-glucuronosyltransferase in human liver. Drug Metab Dispos 39:1538-1545.

Address correspondence to: Koichi Omura, Research Laboratories 2, Fuji Yakuhin Co., Ltd., 636-1 lida-Shinden, Nishi Ward, Saitama City, Saitama 331-0068, Japan. E-mail: k-omura@fujiyakuhin.co.jp 